\title{
Derivation of Mass Independent Quantum Treatment of Phenomenon
}

David Parker

davidparker25301@comcast.net

Follow this and additional works at: http://digitalcommons.wayne.edu/jmasm

Part of the Applied Statistics Commons, Social and Behavioral Sciences Commons, and the Statistical Theory Commons

\section{Recommended Citation}

Parker, David (2010) "Derivation of Mass Independent Quantum Treatment of Phenomenon," Journal of Modern Applied Statistical Methods: Vol. 9 : Iss. 1 , Article 31.

DOI: $10.22237 /$ jmasm/1272688200

Available at: http://digitalcommons.wayne.edu/jmasm/vol9/iss1/31

This Brief Report is brought to you for free and open access by the Open Access Journals at DigitalCommons@WayneState. It has been accepted for inclusion in Journal of Modern Applied Statistical Methods by an authorized editor of DigitalCommons@WayneState. 


\title{
Derivation of Mass Independent Quantum Treatment of Phenomenon
}

\author{
David Parker \\ Oak Park, Michigan
}

The derivation and applications is presented of a spatial variable or spatial radius which is related to the inertia or mass-energy of any quantum body by a Lorentz invariant relation. Mass independent DeBroglie and Schroedinger equations are derived and applied to the resolution of the linguistic incompatibility between quantum theory and the geometrical weak equivalence principle. The equivalence principle is restated in terms of the spatial radius. The gravitational attraction between bodies and the relativistic energy are both presented in terms of the spatial radius follows. The ratio of the gravitational force to the Coulomb force at the Planck scale and is found to equal unity. The annihilation of particle and antiparticle is presented as an interference effect using the spatial radius.

Key words: Mass independent quantum mechanics, sass independent Schroedinger equation, mass independent DeBroglie equation, spatially dependent quantum mechanics.

\section{Introduction}

Quantum mechanics contains a collection of statistical phenomenon known as the waveparticle duality which refers to the unexpected and inexplicable appearance of wave like behavior for a large collection of particles. An example of the bizarre aspect of the waveparticle duality is the appearance of an interference-like pattern after the detection of thousands of electrons which apparently did not interact with each other. The connections between the wave and particle aspects of matter follow the Einstein-DeBroglie relations. However, there is a large conceptual gap between the wave and particle dynamical variables which are utilized in the EinsteinDeBroglie relations (Silverman, 1994).

For example, the wavelength of the wave phenomena associated with matter has a natural physical meaning relative to the wave phenomena but it does not have the same kind of physical relation relative to the particle momentum or inertia. Furthermore, the wavelength is a distance which is the measure of an interval and is a spatial variable which does

David Parker is a private scholar who resides in Michigan. Email him at:

davidparker25301@comcast.net. not directly relate to the momentum or inertial variables. In order to bridge this conceptual gap the dynamical variables of mass and energy which characterize the inertial aspects of matter have been reformulated in terms of the spatial variable which also characterizes the wave aspects of matter. The spatial variable which is introduced in this article is called the spatial radius. The spatial variable also leads to a mass independent formulation of quantum theory and a physical model of the inner structure of the wavelength associated with matter which in turn might give some understanding of the wave-like transfer of inertia by particles. The phrases quantum body or elementary particles refer to particles or bodies in quantum theory, such as photons, bosons, fermions, and their theoretical constituents such as quarks.

The spatial radius is related to the inertia or the mass-energy of any quantum body by a Lorentz invariant relation derived from a generalization of the results of classical experiments. The extension of this concept to any bodies which are not a concern of quantum theory such as astronomical bodies is a work in progress. Physical significance of the spatial radius is found in scattering theory and is discussed below. The spatial radius is also applied to the gravitational attraction between bodies at the Planck scale and the treatment of relativistic energy. 


\section{DAVID PARKER}

Also discussed is an exclusion which follows from the Lorentz invariant form. The spatial radius concept enables the derivation of mass independent three dimensional forms of both the DeBroglie and Schroedinger equations which in turn implies a mass independent quantum treatment of phenomenon and the resolution in principle of the linguistic incompatibility between the existing mass dependent quantum interpretation of nature and the mass independent demands of the geometrical weak equivalence principle (Greenberger \& Overhauser, 1980).

Using the spatial radius, the equivalence principle can be restated as the requirement that the fall of a point particle in the gravitational field of the earth is independent of the spatial radius of the point particle. Verification of the predictions and solutions which are dependent on the spatial radius of the DeBroglie and Schroedinger equations and are derived here can be acquired by comparison with the large body of solutions which have accumulated since the introductions of the original mass-dependent DeBroglie and Schroedinger equations in the previous century.

\section{Derivation of the Spatial Radius}

The spatial radius concept was derived from a generalization of the results of classical experiments which attempted to determine the motion or the self-force of electrons in various fields and implied that the motion of the assumed spherical electrons were dependent on the ratio of the electrostatic energy and the size or radius of the assumed spherical electron with a numerical factor dependent on charge distribution which can be written as $\mathrm{e}^{2} / \mathrm{r}$, which is proportional to the proper energy of the electron where e is the electron charge (esu) and $r$ the radius (Born, 1969). Experimentally the electron was known to be stable, however, the model of an assumed spherical electron was eventually demonstrated by Poincaire to be unstable and so the spherical model was discarded (Heitler, 1953).

Without the spherical model the question arises as to the physical meaning of the electron radius (r) upon which the electron motion was found to be partially dependent. The dependency of the motion on the term $\mathrm{e}^{2} / \mathrm{r}$ was interpreted as implying that the inertial electron mass increased proportionally to $\mathrm{e}^{2} / \mathrm{r}$ and it was also assumed by some physicists that inertial mass of the electron was purely electromagnetic in origin, which seemed possible because the electron mass was so small. This assumption about the electromagnetic origin of the inertial mass implies that the electron radius assumes the value $\mathrm{r}=\mathrm{e}^{2} /\left(\mathrm{m} \times \mathrm{c}^{2}\right)$ and the radius with this particular value became known as the classical electron radius (Born, 1969).

An analysis of the role of the electron charge to the motion in these experiments shows that the role of the electron charge is involved with the inertial mass of the electron. This was interpreted herein as the possibility that the idea of the electron radius could be generalized to all bodies independently as to whether or not the body was charged. Thus, the classical electron radius can be viewed as the spatial radius associated with an electron. The specific form of the generalization follows the form in the Lorentz invariant equation for the relation between the classical electron radius and the electron mass.

The classical electron radius is sometimes presented as a length which is a combination of classical constants but is several orders of magnitude smaller than the average size atom. At present the value of the classical electron radius is probably historical. Contemporary physicists might be surprised however by Max Born's claim that the classical electron radius has an actual physical significance and the simplest phenomena in which it occurs is the scattering of light in the calculation of an 'effective cross scattering' (Born, 1969).

The electron which has the smallest mass can be viewed as the unit of mass, then the mass of any body and the associated spatial radius can be written as a multiple of the electron mass. An indication of the interpretation and the actual physical significance or existence of the spatial radius as a generalization of the classical electron can be found in Max Born's analysis and calculation of the coefficient of scattering of radiation for a free body. Born points out that in the formula for the total energy scattered by a free body I(f) when given an 


\section{DERIVATION OF MASS INDEPENDENT QUANTUM TREATMENT OF PHENOMENON}

initial energy $\mathrm{I}(0) \quad$ is $\mathrm{I}(\mathrm{f})=8 \pi / 3\left(\mathrm{e}^{2} / \mathrm{m} \times \mathrm{c}^{2}\right) \times \mathrm{I}(0)$ which implies that a proton or nucleus scatters some millions of times less than an electron (Born, 1969). The point is that the generalized form of the spatial radius is found in the formula for the energy scattered and also has a physical interpretation. The minute amount of radiation scattered by a large mass means that the phenomena would not be apparent.

The exact expression for the relation between the spatial radius and the mass-energy of the body is $\Phi=\mathrm{R}(\mathrm{M}) \times \mathrm{M}$ and when given the energy, $\mathrm{E}$, of the body then $\mathrm{E} \times \mathrm{R}(\mathrm{E})=\left(\Phi \times \mathrm{c}^{2}\right)$ with the important result that $\mathrm{R}(\mathrm{E})=\mathrm{R}(\mathrm{M})$ for any quantum body. The expressions for $\mathrm{R}(\mathrm{M})$ and $\mathrm{R}(\mathrm{E})$ are Lorentz invariant. From $\mathrm{e}^{2}=\alpha \times \hbar \times \mathrm{c}$ the constant $\Phi$ can also be written as $\Phi=(\alpha \times \hbar) / \mathrm{c}$ where $\alpha$ is the fine structure constant and $\hbar$ is Planck's reduced constant. The discussion of relativistic energy will result in the spatial radius having either a positive or negative value $( \pm R(M)$ or $\pm R(E))$ with the negative value referring to the antiparticle. Objection to the generalization of the properties of a charged electron to uncharged bodies should be reconsidered in terms of the quark theory.

The exclusion mentioned above refers to physical variables of any quantum body which originates within the Lorentz invariant definition of the spatial radius. The exclusion is that the spatial radius and the set of all physical variables which, during a finite time interval, describe the state of the body such as the mass, energy, etc. always have non-zero finite values. The sign of each variable is the sign of the spatial radius. The exclusion of zero-valued variables requires that any change of the sign of the spatial radius must be discontinuous which is consistent with the spirit of quantum theory. An example of a transition which is clearly discontinuous is mentioned by Heitler where he points out that a rapidly varying external field can cause a transition of an electron with positive energy to a negative energy state with positive charge (positron) (Heitler,1953). The assumption that all variables assume the sign of the spatial radius implies that the mass of anti-particles becomes negative does not necessarily refer to the inertial mass of the anti-particle. However, it is believed that negative inertial mass will behave or fall exactly like positive inertial mass in a weak gravitational field.

Algebra of the Spatial Radius

Given mass $\mathrm{M}$ and mass $\mathrm{N}$, then

$$
\begin{gathered}
\mathrm{M} \times \mathrm{N}=\frac{\Phi^{2}}{\mathrm{R}(\mathrm{M}) \times \mathrm{R}(\mathrm{N})}, \\
\mathrm{M}+\mathrm{N}=\frac{\Phi \times(\mathrm{R}(\mathrm{N})+\mathrm{R}(\mathrm{M}))}{\mathrm{R}(\mathrm{M}) \times \mathrm{R}(\mathrm{N})}
\end{gathered}
$$

and

$$
\frac{\mathrm{M}}{\mathrm{N}}=\frac{\mathrm{R}(\mathrm{N})}{\mathrm{R}(\mathrm{M})} .
$$

Thus, the reduced mass $(\mu)$ is:

$$
\mu=\frac{\Phi}{\mathrm{R}(\mathrm{M})+\mathrm{R}(\mathrm{N})} .
$$

\section{Methodology}

Derivation of the Mass Independent and Spatially Dependent DeBroglie Equation

The mass independent form of the DeBroglie equation for the wavelength of the wave phenomena associated with any quantum body is derived by first solving for the body mass in terms of the spatial radius and substituting for the mass in the momentum. Then the DeBroglie equation becomes:

$$
\begin{aligned}
\lambda & =\mathrm{h} /(\mathrm{M} \times \mathrm{v}) \\
& =(\mathrm{h} \times \mathrm{R}(\mathrm{M})) /(\Phi \times \mathrm{v}) \\
& =(\mathrm{h} \times \mathrm{R}(\mathrm{M}) \times \mathrm{c}) /(\alpha \times \hbar \times \mathrm{v}) \\
& =(2 \pi \times \mathrm{R}(\mathrm{M}) \times \mathrm{c}) /(\alpha \times \mathrm{v}) \\
& =\mathrm{h} / \mathrm{p}
\end{aligned}
$$

using $\Phi=\alpha \times \hbar / \mathrm{c}$, where $\alpha$ is the fine structure constant and $\hbar$ is Planck's constant in reduced form. For the spatial radius when given 


\section{DAVID PARKER}

as a function of the energy $R(E)$ the wavelength has the form $\lambda=(2 \pi \times R(E)) / \alpha$.

$$
\begin{gathered}
\lambda=\left[\frac{1}{\alpha}\right] \times[2 \pi \times \mathrm{R}(\mathrm{M})] \times\left[\frac{\mathrm{c}}{\mathrm{v}}\right] \\
\lambda=\frac{[2 \pi \times \mathrm{R}(\mathrm{E})]}{\alpha}
\end{gathered}
$$

The arrangement of the wavelength in equations $1 \mathrm{a}$ and $1 \mathrm{~b}$ is interpreted here as being the fine structure of the wavelength where the quantity $1 / \alpha \approx 137$ is the number of spheres of size $2 \pi \times R(M)$ which comprise the wavelength and are also arranged in a line in the direction of motion of the body. Each sphere has an inertial aspect in the direction of motion of the body and the sum of the spheres equals the total inertia in the direction of the motion. The point is that the total inertia of a body is not transmitted in one whole impulse but appears to be transferred in a linearly quantized manner like a wave in the direction of motion. From this assumption it appears that the inertial aspects are also related to the fine structure constant $\alpha$. The component of the wavelength in the direction of motion varies relativistically; this is consistent with the correlation of particle aspects at high energies.

Derivation of the Wavelength from the Uncertainty Principle

The spatially dependent wavelength can be easily derived from the calculation of the product of the uncertainties in momentum and position $\Delta \mathrm{P} \times \Delta \mathrm{x} \geq \mathrm{h}$ with the substitution of the spatial radius for the mass in the momentum $\mathrm{P}=\mathrm{M} \times \mathrm{v}$ and by assuming that the wavelength $\lambda=\Delta \mathrm{x}$.

Derivation of Non-Relativistic Mass Independent Schroedinger Equation

The simplest derivation is obtained by substituting the spatial radius for the mass into the co-efficient of the second derivative of the wave function with respect to space in the original non-relativistic mass-dependent Schroedinger equation. The resulting spatially dependent Schroedinger implies a spatial interpretation of quantum theory.

$$
\begin{aligned}
& \left\{\left[\frac{\neg \hbar}{2 \alpha}\right] \times[\mathrm{R}(\mathrm{M})] \times[\mathrm{c}] \times\left[\frac{\partial^{2} \Psi(\mathrm{x}, \mathrm{t})}{\partial \mathrm{x}^{2}}\right]\right\}+\{[\mathrm{V}(\mathrm{x}, \mathrm{t})] \times[\Psi(\mathrm{x}, \mathrm{t})]\} \\
& =\left\{[\mathrm{ix \hbar}] \times\left[\frac{\partial \psi(\mathrm{x}, \mathrm{t})}{\partial \mathrm{t}}\right]\right\}
\end{aligned}
$$

\section{Gravitational Attraction between Bodies}

The classical gravitational attraction between bodies in terms of the spatial radius is derived with the substitution of the spatial radius for the mass of each body. As the scale of events approaches the Planck scale it is generally believed that the ratio of the gravitational force to the Coulomb force approaches unity. The ratio of the gravitational and Coulomb forces in terms of the spatial radius is given in equation $3 \mathrm{~b}$, which is the gravitational force between two bodies each with the Planck mass, $\dot{\mathrm{m}}$, separated by the distance $\mathrm{S}$. The Coulomb force is between two unit charges separated by the distance $\mathrm{S}$. Note that $\dot{L} \times \alpha=R(\dot{\mathrm{m}})$ where $\dot{\mathrm{L}}$ is the Planck length and $\mathrm{R}(\dot{\mathrm{m}})$ is the spatial radius of the Planck mass. It might be possible with the use of the spatial radius to smooth out the violent fluctuations at the Planck length. The gravitational force between two bodies with mass $\mathrm{M}$ and $\mathrm{N}$ is:

$$
\begin{aligned}
\mathrm{F} & =\frac{-\left[\Phi^{2} \times \mathrm{G}\right]}{\left[\mathrm{R}(\mathrm{M}) \times \mathrm{R}(\mathrm{N}) \times \mathrm{S}^{2}\right]} \\
\frac{\mathrm{F}(\mathrm{GRAV})}{\mathrm{F}(\mathrm{COUL})} & =\frac{\left[\left(\mathrm{G} \times \Phi^{2} \times \alpha \times \kappa\right)\right] /\left[\mathrm{R}^{2}(\dot{\mathrm{m}}) \times \mathrm{S}^{2}\right]}{\kappa \times \mathrm{e}^{2} / \mathrm{S}^{2}} \\
& =1
\end{aligned}
$$

where $R(\dot{m})$ is the spatial radius of the Planck mass and $\mathrm{K}$ is the constant of proportionality in Coulomb's law.

$$
\mathrm{F}(\mathrm{GRAV}) \times[(\alpha \times \kappa)]=\mathrm{F}(\mathrm{COUL})
$$

or

$$
\mathrm{F}(\mathrm{GRAV})=(\hbar \times \mathrm{c}) / \mathrm{S}^{2}
$$




\section{DERIVATION OF MASS INDEPENDENT QUANTUM TREATMENT OF PHENOMENON}

\section{Relativistic Energy and the Spatial Radius}

If the relativistic energy in terms of the spatial radius is calculated for a body with a given rest mass $\mathrm{m}(\mathrm{o})$ and velocity there will be a negative and a positive valued solution. The negative value is the energy of the anti-particle.

$$
\begin{aligned}
\mathrm{E}(+) & =\Phi \times \mathrm{c}^{2} / \mathrm{R}(\mathrm{m}(\mathrm{o})) \\
& =\mathrm{e}^{2} / \mathrm{R}(\mathrm{m}(\mathrm{o})) \\
\mathrm{E}(\neg) & =\neg \Phi \times \mathrm{c}^{2} / \mathrm{R}(\mathrm{m}(\mathrm{o})) \\
& =\neg \mathrm{e}^{2} / \mathrm{R}(\mathrm{m}(\mathrm{o}))
\end{aligned}
$$

Interference Effects and Annihilation of Particles

When particle and anti-particle interact and mutually annihilate they generally form a pair or trio of photons. The assumption here is that the annihilation is caused by the destructive interference of the out of phase wave phenomenon associated with the wave aspects of particle and anti-particle which are related to the spatial radius.

The sign of the spatial radius refers to the in or out aspect of the particle's wave phenomena. Interference phenomenon requires a dimensional representation for the superposition of the waves, which is regarded as motivation for the assumption of three dimensionality. Interference effects are also assumed to have a role in the exclusion principle in the repositioning of the orbital electrons.

The Relation of the Planck Scale to All Quantum Bodies

The Planck scale is a set of physical variables formed from various combinations of the gravitational constant, Planck's constant (reduced) and light speed. Given any particle $\mathrm{P}$ with finite non-zero mass $M$ and radius $R(M)$ it follows that $\mathrm{M} \times \mathrm{R}(\mathrm{M})=\mathrm{m}(\mathrm{e}) \times \mathrm{R}(\mathrm{e})=\Phi$ where $m(e)$ is the electron mass and $R(e)$ is the electron radius. The product of the fine structure constant, the Planck mass and the Planck length is equal to $[\dot{\mathrm{m}} \times \dot{\mathrm{L}} \times \alpha]=\Phi$. Then the spatial radius concept implies that the Planck mass and the Planck length are related to the mass and radius of any quantum body. It is also implied that the Planck energy has a relation to the energy all of the quantum bodies.

Conclusion and Brief Model of Quantum Bodies

All quantum bodies are assumed to exist as three dimensional structures during their lifetime. The axis of any quantum particle in the instantaneous direction of motion is called the relativistic axis. The axes normal to the relativistic axes are called the virtual axes. The interactions that occur on the relativistic axis are generally time dependent and the interactions on the virtual axes are generally independent of time. Interference effects and repositioning is assumed to occur along the virtual axes. Entanglement phenomena, which occur with particles which travel at light speed, are also assumed to occur along the virtual axes. A description of the interactions that occur along the virtual axes is assumed to be given by the Feynman path integral interpretation, which can be considered time independent at each point of a trajectory.

The question of time dependence might be tested by a variation of the experiment where the emitter is shut down after a single electron emission and allowed to cool and then emitting the second electron and cooling off and so on. The spatial radius always has a non-zero value on all three axes of the body. The inertial variables of the body are distributed along the spatial radius and therefore vary statistically as a function of the statistical variation of the spatial radius. The shape of the particle at any particular time is the three dimensional envelope formed from the tips of the spatial radii. In a minimal sense the spatial radius is a fresh approach and is a unique interpretation of quantum theory.

The application of a radius of a particle was suggested by DeBroglie as a way to avoid the pitfalls of infinite self-energies which occur with point particles (DeBroglie, 1960). Verification of the spatial radius might be obtained from scattering experiments. It may be both interesting and useful to repeat the classical experiments which resulted in size dependent behavior for an electron. Time is regarded here as a secondary variable which is dependent on motion. 


\section{DAVID PARKER}

Acknowledgements

For my parents and Lawrence Resnick. The author also thanks Grant R. Gerhart and Richard Felch.

\section{References}

Born, M. (1969). Atomic physics. Delaware: Dover Publications.

DeBroglie, L. (1960). Non-linear waver mechanics a causal interpretation. London: Elsevier Publishing Company.
Greenberger, D. M., \& Overhauser, A. W. (1980). The role of gravity in quantum theory. Scientific American, 242(5), 66-76.

Heitler, W. (1953). The quantum theory of radiation. DE: Dover Publications.

Silverman, M. P. (1994). More than one mystery. New York: Springer-Verlag. 